\title{
The efficacy of repetitive transcranial magnetic stimulation in treating patients with chronic daily headache
}

\author{
Ann Ali AbdElkader', Ebtesam Mohammed Fahmy², Ayat Allah Farouk Ahmad', Amira A. Labib', \\ Leqaa Ali El-Mekkawy ${ }^{1}$ and Shaimaa shaheen Mohammed Abd El Razek ${ }^{2^{*}}$ (D)
}

\begin{abstract}
Background: Headache is the most common pain disorder, affecting around $66 \%$ of the global population. This study aimed to investigate the efficacy of high-frequency repetitive transcranial magnetic stimulation (rTMS) in treating patients with primary chronic daily headaches (chronic tension-type headache and chronic migraine).

Methods: Twenty-seven patients participated in the study, divided into 2 groups: a study group (16 patients) and a control group (11 patients). Treatment consisted of 12 high-frequency $(5 \mathrm{~Hz})$ real rTMS sessions, delivered over the left dorsolateral prefrontal cortex (DLPFC), whereas sham rTMS was used for the control group.

Results: Patients of the study group, after real rTMS stimulation, showed a high statistically significant reduction of the measured headache parameters compared to the control group ( $P$ value $<0.001$ ), and the percentage of improvement was $94.5 \%$. No significant reduction of headache parameters, after sham rTMS stimulation, was observed in the control group ( $P$ value $>0.05$ ) and the percentage of improvement was $7.9 \%$.

Conclusion: High-frequency rTMS is effective in reducing chronic tension headaches and chronic migraines. This finding runs with the approval of the suggested role of DLPFC in pain control. This might open opinions for new treatment strategies in tension-type headache and migraine prevention.
\end{abstract}

Keywords: Repetitive trans-cranial magnetic stimulation, Chronic daily headache, Tension headache, Migraine

\section{Introduction}

Chronic daily headache $(\mathrm{CDH})$ is defined as headaches that occur for a minimum of 15 days in a month, for at least 3 months, for at least $4 \mathrm{~h}$ in a day untreated. Primary $\mathrm{CDH}$ includes chronic tension-type headache, hemicrania continua, new daily persistent headache, and chronic migraine $(\mathrm{CM})$ [1].

Chronic tension-type headache $(\mathrm{CTTH})$ is the most common form of headaches. It represents approximately $80 \%$ of all headache diagnoses [2]. Clinically, CTTH has no specific features, a low-level headache that is never severe, and never has migrainous characters. Patients

\footnotetext{
* Correspondence: Shaheen.shaimaa@yahoo.com

${ }^{2}$ Department of Neurology, Faculty of Medicine, Cairo University, Cairo, Egypt Full list of author information is available at the end of the article
}

usually have either photophobia, phonophobia, or mild nausea or none of these. The pain is symptomatized as aching or pressure and feeling as if the head has a tight band around it $[1-3]$.

Migraine is a prevalent neurological disorder. Reports have declared that $18 \%$ of men and $43 \%$ of women experience a migraine at a certain era of their lives $[4,5]$. Migraine is often described as recurrent, pulsating or throbbing, moderate to severe, and usually unilateral pain that occurs for $4-72 \mathrm{~h}$, with complete relief between the episodes (episodic). The headache is associated with nausea, vomiting and/or photophobia, phonophobia, or olfactory disorders. Visual aura, forms of zigzag lines or wide scintillating scotoma, is the most common; also, unilateral sensory abnormalities and/or disorders of deglutition may occur [3]. 
Transcranial magnetic stimulation (TMS) is a noninvasive and safe way to transiently modify the brain cortical excitability, through applying brief magnetic pulse(s) over the head. TMS was able to suppress cortical spreading depression in animal model experiments, proposing potential therapeutic implications especially in migraine with aura attacks [6].

Repetitive transcranial magnetic stimulation (rTMS) is defined by the number of pulses in a second or frequency in Hertz $(\mathrm{Hz})$. According to the frequency it is divided into "low-frequency" (slow) rTMS, using $1 \mathrm{~Hz}$ or less, and "high frequency" (fast) rTMS, using s frequency higher than $1 \mathrm{~Hz}$ (ranging between 5 and $25 \mathrm{~Hz}$ ). Another variable of stimulation is the intensity expressed as the percentage of individual resting motor threshold. Repetitive TMS can induce long-lasting changes in the underlying cortex [7].

The prefrontal cortex is the area that may be especially beneficial in modulating the sentimental, attentional, and affective aspects of pain [8]. Brighina and colleagues [9] have elucidated analgesic effects with prefrontal cortex rTMS. The role of the left prefrontal cortex in pain modification is unclear; however, there are theories to support the idea that left prefrontal stimulation exerts active control on pain sensation by modulating corticosubcortical and cortico-cortical tracts [10].

This study was designed to assess the efficacy of rTMS in the treatment of patients with chronic tension headache and chronic migraine, aiming at raising a new treatment regimen, and thus elevating the quality of the daily life of such patients. This data has been presented previously [11].

\section{Methods}

This is a randomized controlled single-blinded study carried out on 27 patients ( 22 females and 5 males) having chronic daily headaches according to the International Headache Society (IHS) [12].

All patients were informed about the aim and procedures of the study, and an informed written consent has been obtained from the patients before the study and a proper explanation of possible complications (like hearing loss which is prevented by using a protective hearing set) was done.

Patients were divided into 2 groups: the study group which included 16 patients who received real $(5 \mathrm{~Hz})$ rTMS and the control group which included 11 patients who received sham rTMS. Patients in both groups are adults; their age is above the age of 18 years old. They suffered from chronic daily headaches (chronic tension-type headache and chronic migraine) with no improvement on medications for a minimum period of 3 months.
Patients were collected from the out-patient clinic, in the period from December 2012 to September 2013 under the supervision of the neurologist in the outpatient clinic.

Patients with abnormal computed tomography (CT) or magnetic resonance imaging (MRI) of the brain or with abnormal conventional EEG were excluded from the study. Patients in this study are not depressed as Hamilton depression scale has been done for them to exclude depression (their score is 0-7 to exclude depression). Patients with other subtypes of primary headache and secondary causes of headache, epilepsy or family history of epilepsy, pregnant females, and subjects with a pacemaker, intracranial metal objects, and artificial metal tooth were excluded also from the study.

Patients were subjected to assessment procedures in the form of a clinical assessment including careful history taking and complete neurological examination. Headache assessment scale; after the first visit, patients were given a diary to rate headache parameters before starting the first session and after finishing all the treatment sessions. The scale included the duration of headaches, frequency of the episodes per month, pain severity, detected on a scale of 4 points (from 0 to 3) with 0 (no pain), 1 (mild pain), 2 (moderate pain), and 3 (severe pain) and headache index (frequency $\times$ intensity) [9]. The scale was applied for all patients prior to and after the treatment sessions immediately, and the patients had been followed for a month from the application of rTMS to confirm consistency of results. Routine laboratory tests included complete blood count (CBC), fasting and $2 \mathrm{~h}$ postprandial blood sugar level, liver and kidney function tests, erythrocyte sedimentation rate (ESR), and collagen vascular profile. Radiological assessment was done such as CT (computed tomography, Toshiba, Aquilion; 64 slices, Japan, 2005) and/or MRI of the brain (magnetic resonance imaging, Philips, achiva and intera; 1.5 Tesla, Holland, 2003) to exclude secondary causes of headache.

Treatment procedures: repetitive trans-cranial magnetic stimulation: A high-frequency repetitive transcranial magnetic stimulator (MagStim Rapid magnetic stimulator, Magstim Company, Whitland, Wales, UK), connected with a figure-of-eight coil with a diameter of $70 \mathrm{~mm}$ was used.

The motor threshold (MT) was specified for all subjects before the first session, using single-pulse stimulation over the primary motor cortex at the hot spot of the first dorsal interosseous (FDI) muscle, using a standard EMG machine and surface electrodes. The MT was defined as the lowest intensity required to provoke a motor-evoked potential in 50\% of successive trials or to induce a visible movement 
of the thumb, wrist, or fingers in at least half of 10 stimulations in a fully relaxed muscle [9] . The figure-of-eight coil was placed tangentially over the left DLPFC cortex which was applied on the scalp, 5 $\mathrm{cm}$ anterior to the hot spot for the FDI muscle [13], with its handle pointing posteriorly and placed parallel to a mid-sagittal axis of the head.

The study group got 12 sessions of high-frequency rTMS, received every other day, with the exclusion of weekends for 4 sequential weeks. Each rTMS session entailed 10 trains of 10 stimulation pulses, with a train duration of $2 \mathrm{~s}$, separated by a $30-\mathrm{s}$ period, applied at $5-\mathrm{Hz}$ frequency, and $90 \%$ of the MT intensity over left DLPFC [9].

The control group got sham stimulation by turning the coil $90^{\circ}$ from the scalp over the same area of the skull and the same intensity and protocol as real rTMS. This technique produced a sound similar to the active stimulation and some somatic sensations with negligible direct cortical sequalae [14].

Statistical Methods: MedCalc $^{\circ}$ version 12.3.0.0 program "Ostend, Belgium" was used for calculations of sample size, statistical calculator based on 95\% confidence interval and power of the study $80 \%$ with $\alpha$ error $5 \%$. The previous study [15] showed that there was a mean of post treatment at headache index in the study group of $3.06 \pm 2.999$ compared to the control group of $52.18 \pm 18.819$, with $p$ value $<0.001$ highly significant. So it can be relied upon in this study, based on this assumption, that the sample size was calculated according to these values produced in a minimal sample size of 27 cases that were enough to find such a difference, subdivided into two groups, study group $(n=16)$ and control group $(n=11)$.

Data were processed using SPSS in statistical package version 20 (SPSS Inc., Chicago, IL). Numerical data were analyzed as mean and standard deviation or median and range accordingly. Qualitative data were analyzed as frequency and percentage. Chi-square test (Fisher's exact test) was utilized to test the relation between qualitative variables. For quantitative data, in comparing 2 groups, either Student $t$ test or MannWhitney test (non-parametric $t$ test) was used accordingly. Paired $T$ test was utilized on comparing to show the effect of intervention between pre and post clinical assessment. The percent of improvement was calculated for study and control groups by (HI before treatment-HI after treatment, divided by HI before treatment, multiplied by 100). Bivariate correlation analysis was carried out to assess treatment response with age, headache duration, frequency, and intensity. Pearson correlation coefficient was utilized to assess treatment response with a type of headache. An Independent sample $t$ test was also used. A $p$ value $\leq 0.05$ was considered significant.

\section{Results}

\section{General characteristics of studied groups}

The study group included 16 patients (13 females and 3 males), with a mean age of $34.19 \pm 9.738$ years. The duration of illness ranged from 3 to 144 months with a mean of $22 \pm 36.13$ months. The control group included 11 patients ( 9 females and 2 males), with a mean age of $33.91 \pm 8.526$ years. The duration of illness ranged from 3 to 144 months with a mean of $31.36 \pm 42.37$ months. Both groups were matched regarding mean age, gender, and duration of illness ( $P$ value $>0.05)$.

The study group included 16 patients, $50 \%$ of them had chronic migraines and 50\% had a chronic tension headache. The control group included 11 patients, $54.5 \%$ had chronic migraine, and the remaining $45.5 \%$ had a chronic tension headache.

Headache parameters before treatment in both groups are represented in Table 1 . No statistically significant difference was noted between the study and control groups regarding headache frequency, intensity, or index before treatment $(P=0.39,0.88,0.69)$ respectively.

Headache parameters after treatment were represented;

Within groups: A high statistically significant decrease in headache frequency, intensity, and index was observed in the study group patients after treatment $(P$ value < 0.001 ). The percentage of improvement was $94.5 \%$ (percent of patients within this group who show improvement in frequency and intensity of headache), which indicates a significant improvement of the subjects of this group after treatment (Tables 2 and 3). The same degree of improvement was found in chronic migrainous as well as chronic tension headache patients.

No significant reduction in headache parameters was observed in patients of the control group $(P$ value > $0.05)$. The percentage of improvement was $7.9 \%$, which indicates a non-significant improvement of these patients after treatment (Tables 2 and 4).

Between groups: A high statistically significant decrease regarding headache frequency, intensity, and index was observed after treatment in the study group on being compared to the control group $(P=0.001)$ (Table 5).

No significant correlation was detected between the response to rTMS treatment with the age of the patients, duration of illness, headache frequency, or headache intensity in the study group $(P=0.27, r=-0.29),(P=$ 0.72, $r=0.09),(P=0.98, r=0.001),(P=0.72, r=0.09$, respectively).

\section{Discussion}

Headache disorders are among the most dominant central nervous system diseases affecting $66 \%$ of the population worldwide and thus considered as one of the major health issues. Seventy-five percent of patients report failure carrying out their work during the attack, and 50\% 
Table 1 Comparison of pre-treatment headache parameters between study and control groups

\begin{tabular}{|c|c|c|c|c|}
\hline \multirow[t]{2}{*}{ Headache intensity } & \multicolumn{2}{|l|}{ Study group } & \multicolumn{2}{|l|}{ Control group } \\
\hline & Number & Percentage & Number & Percentage \\
\hline Mild & 1 & 6.3 & 0 & 0 \\
\hline Moderate & 7 & 43.8 & 4 & 36.4 \\
\hline Severe & 8 & 50 & 7 & 63.6 \\
\hline Total & 16 & 100 & 11 & 100 \\
\hline$P$ value & 0.39 & & & \\
\hline \multirow[t]{2}{*}{ Headache frequency } & Mean \pm SD & Range & Mean \pm SD & Range \\
\hline & $23.50 \pm 6.782$ & $15-30$ & $23.09 \pm 7.14$ & $15-30$ \\
\hline$P$ value & 0.88 & & & \\
\hline Headache index & $58.00 \pm 22.891$ & $15-90$ & $61.64 \pm 24.42$ & 30-90 \\
\hline$P$ value & 0.69 & & & \\
\hline
\end{tabular}

$S D$ standard deviation

require the aid of a family member, with extensive impact on their quality of life [3].

In this study, the age of the patients ranged from 20 to 45 years, as migraine commonly affects the age range of 25-55 years [16], as for CTTH is in the age range of 30 to 39 years [17]. Twenty-two patients were females (81.5\%) and 5 were males (18.5\%), with male to female ratio 1:4.4. This went with previous studies that stated that $\mathrm{CDH}$ is more common in females than in males [16-19]. This distribution of age and gender-specific prevalence rates of migraine might indicate the involvement of hormonal factors in the development of migraine.

In the current study, a high statistically significant decrease regarding headache intensity, frequency, and index was found in the group who received real rTMS. These results went in accordance with Brighina and colleagues [9] who concluded that treatment with rTMS was of extreme benefit in ameliorating headache frequency and headache index.

The precise technique by which rapid rate magnetic stimulation over the left frontal cortex ameliorates chronic headache is still unclear. Previous studies noted that rTMS treatment at high frequencies was capable of retrieving normal or quite normal levels of DLPFC activation [20].
Both migraine and chronic pain have features in common that may depend on fronto-limbic sensitization. In chronic migraine, pain becomes quite independent from operating triggers and is commonly associated with "limbic" symptoms, such as sleep disorders, fatigue impaired memory and concentration, and reduced libido. So, it is possible that DLPFC activation might reset or minimize frontolimbic dysfunction, causing improvement of the clinical condition. Indeed, although magnetic stimulation was restricted to the prefrontal area, remote influences of rTMS (network effects) may also spread to faraway interconnected areas [21].

Repetitive TMS can enhance long-lasting plastic modifications, causing a functional reorganization of the underlying cortex. Even single-pulse TMS can involve many brain networks that are linked functionally to the visual system, which might be responsible for its effects in migraine [22].

In this study, high-frequency rTMS utilized on the left DLPFC was of great benefit in treating CTTH and CM patients. This finding went in accordance with Lorenz and colleagues [10] who stated that DLPFC seems to have an inhibitory effect on pain perception, by acting as a negative modulation of central supra-spinal pain tracts.

Table 2 Comparison of headache intensity before and after treatment within the study and control groups

\begin{tabular}{|c|c|c|c|c|c|c|c|c|}
\hline \multirow{3}{*}{$\begin{array}{l}\text { Headache } \\
\text { intensity }\end{array}$} & \multicolumn{4}{|c|}{ Study group (16) } & \multicolumn{4}{|c|}{ Control group (11) } \\
\hline & \multicolumn{2}{|c|}{ Pre-treatment } & \multicolumn{2}{|c|}{ Post treatment } & \multicolumn{2}{|c|}{ Pre-treatment } & \multicolumn{2}{|c|}{ Post treatment } \\
\hline & $N$ & $\%$ & $n$ & $\%$ & $n$ & $\%$ & $n$ & $\%$ \\
\hline Mild & 1 & 6.3 & 14 & 87.5 & 0 & 0 & 0 & 0 \\
\hline Moderate & 7 & 43.8 & 2 & 12.5 & 4 & 36.4 & 4 & 36.4 \\
\hline Severe & 8 & 50 & 0 & 0 & 7 & 63.6 & 7 & 63.6 \\
\hline$P$ value & \multicolumn{4}{|c|}{$<0.001^{*}$} & \multicolumn{2}{|c|}{$>0.05$} & & \\
\hline
\end{tabular}

$n$ number

*Highly significant 
Table 3 Comparison of headache frequency and index before and after treatment within the study group

\begin{tabular}{|c|c|c|c|c|}
\hline & \multicolumn{4}{|l|}{ Study group } \\
\hline & \multicolumn{2}{|c|}{ Headache index } & \multicolumn{2}{|c|}{ Headache frequency } \\
\hline & Pre-treatment & Post treatment & Pre-treatment & Post treatment \\
\hline Mean \pm S.D & $58.0 \pm 22.891$ & $3.06 \pm 2.999$ & $23.50 \pm 6.782$ & $3.19 \pm 1.328$ \\
\hline Range & $15-90$ & $0-10$ & $15-30$ & $1-5$ \\
\hline$P$ value & $<0.001^{*}$ & & $<0.001 *$ & \\
\hline
\end{tabular}

SD standard deviation

*Highly significant

Moreover, DLPFC and the anatomically and physiologically attached limbic cortex have been supposed to be of extreme importance in the pathophysiology of many chronic neurobehavioral conditions, as for example, addiction, depression, bipolar disorder, and migraine.

In the study group, the improvement of patients with CTTH might be the resultant of the alleviation of pain caused by left DLPFC excitation. This finding went in agreement with Brighina and colleagues [23]who reported that $5 \mathrm{~Hz}$ rTMS stimulation at the left DLPFC at 10 or 20 min after capsaicin application over the dorsal surface of the right or left hands on a square area of $2 \times$ $2 \mathrm{~cm}$ lead to a significant decrease in spontaneous pain in both hands [22]. While stimulation of the right DLPF $\mathrm{C}$ with the same parameters revealed no significant influence on pain parameters. According to these results, stimulation of left DLPFC to cause a bilateral control on pain system, supporting the critical antinociceptive role of such area.

In the current study, patients with chronic migraines who were candidates for real rTMS showed a significant decrease of headache measures after treatment. This was consistent with the study of Clarke and colleagues [24] which was carried out on 42 patients with migraine treated with rTMS $(n=23)$ or placebo $(n=19)$. They found that about $69 \%$ of the subjects was cured from headache within $2 \mathrm{~h}$ of active treatment, compared with $48 \%$ in the placebo group. Misra and colleagues [25] also found that 3 sessions of high frequency $(10 \mathrm{~Hz})$ rTMS delivered to left frontal cortex every other day, comprising 600 pulses in 10 trains was beneficial for migraine prophylaxis. About $98 \%$ of patients had more than $50 \%$ decrease of occurrence of the attacks at the end and 1 week after rTMS, and the improvement continued till the fourth week in $80.4 \%$ cases. In a study of Kumar and colleagues [26] 14 patients with migraine and unipolar depression were included in the study with improvement in MIDAS and in Hamilton depression score after 15 sessions of rTMS over the DLFC.

Stimulation of the left DLFC was found to reduce the capsaicin-induced pain over both hands not only the right hand indicating that the left DLFC has an antinociceptive effect on both sides. The factor that enhances the effect of the frontal lobe in migraineurs is the impairment in frontal lobe neuropsychological tests in patients with migraine and the reversal of hypometabolism that is found in the frontal lobe after rTMS make it possible to improve the patient condition [25].

A new study done by Kumar and colleagues [27] showed that stimulation of the left frontal cortex in patients with chronic migraine reduces the frequency and intensity of headache. This previous finding is explained by that the motor cortex is part of the nociceptive circuit [26].

In the present study, patients who got sham rTMS stimulation expressed no evident improvement regarding headache frequency, intensity, and index with a small percentage of improvement (7.9\%). These findings went in accordance with Brighina and colleagues [11], who stated that placebo rTMS leads only to a slight reduction in the frequency of episodes and headache index with no significant improvement of the patients.

\section{Conclusion}

In view of the outcome of the current study, it could be concluded that high-frequency rTMS is effective in the

Table 4 Comparison of headache frequency and index before and after treatment within the control group

\begin{tabular}{|c|c|c|c|c|}
\hline & \multicolumn{4}{|l|}{ Control group } \\
\hline & \multicolumn{2}{|l|}{ Headache index } & \multicolumn{2}{|c|}{ Headache frequency } \\
\hline & Pre-treatment & Post treatment & Pre-treatment & Post treatment \\
\hline Mean \pm S.D & $61.64 \pm 24.422$ & $52.18 \pm 18.819$ & $23.09 \pm 7.148$ & $21.45 \pm 6.729$ \\
\hline Range & 30-90 & $26-90$ & $15-30$ & $13-30$ \\
\hline$P$ value & $>0.05$ & & $>0.05$ & \\
\hline
\end{tabular}

$S D$ standard deviation 
Table 5 Comparison of post treatment headache parameters between the study and control groups

\begin{tabular}{llll}
\hline & $\begin{array}{l}\text { Study group } \\
\text { Mean } \pm \text { SD }\end{array}$ & $\begin{array}{l}\text { Control group } \\
\text { Mean } \pm \text { SD }\end{array}$ & $\begin{array}{l}\boldsymbol{P} \\
\text { value }\end{array}$ \\
\hline Headache frequency & $3.19 \pm 1.328$ & $21.45 \pm 6.729$ & $\mathbf{0 . 0 0 1 *}$ \\
Headache intensity & $0.81 \pm .655$ & $2.45 \pm 0.522$ & $\mathbf{0 . 0 0 1 *}$ \\
Headache index & $3.06 \pm 2.999$ & $52.18 \pm 18.819$ & $\mathbf{0 . 0 0 1 *}$ \\
\hline
\end{tabular}

"Highly significant

treatment and prophylaxis of patients with chronic tension headache and chronic migraine. So, it is recommended to consider high-frequency rTMS as a treatment option for patients with chronic primary daily headaches, especially those refractory to medication.

\section{Limitation}

In our study, there is some limitation from which is the few number of patients incriminated in the study. The control group at least should be the same number of cases or even higher. The patient should be followed for a longer duration to confirm the consistency of our results.

\section{Abbreviations}

$\mathrm{CDH}$ : Chronic daily headache; CM: Chronic migraine; $\mathrm{CTTH}$ : Chronic tensiontype headache; CT: Computed tomography; CBC: Complete blood count; DLPFC: Dorsolateral prefrontal cortex; FDI: First dorsal interosseous; IHS: International Headache Society; MRI: Magnetic resonance imaging; MT: Motor threshold; rTMS: Repetitive transcranial magnetic stimulation; TMS: Trans-cranial magnetic stimulation

\section{Acknowledgements}

Not applicable.

\section{Authors' contributions}

We confirm that A.A.A contributes in the conception and design of the study and follow up the study. E. contributes in the conception and design of the study and follow up the study. A. A. F. has done substantial contributions to the conception and design of the study and in interpretation of data. A. A. L. contributes in analysis and interpretation of data and drafting the article. L. contributes in acquisition of data, analysis and interpretation of data and drafting the article. Sh. Contributes in acquisition of data, analysis and interpretation of data and drafting the article. All authors read and approved the manuscript.

\section{Funding}

No funding received.

\section{Availability of data and materials}

The datasets generated and/or analyzed during the current study are not publicly available due to current Cairo University regulations and Egyptian legislation but are available from the corresponding author on reasonable request and after institutional approval.

\section{Ethics approval and consent to participate}

It was approved by ethical committee of the neurology department, Cairo University on December 2012. No available number.

A written consent was taken from all patients to undergo therapy by this machine and consent for the probable drawbacks that may happen.

\section{Consent for publication}

Not applicable.

\section{Competing interests}

The authors declare that they have no competing interests.

\section{Author details \\ ${ }^{1}$ Clinical Neurophysiology Unit, Faculty of Medicine, Cairo University, Cairo, Egypt. ${ }^{2}$ Department of Neurology, Faculty of Medicine, Cairo University, Cairo, Egypt.}

Received: 14 July 2020 Accepted: 20 January 2021

Published online: 08 February 2021

\section{References}

1. Tepper SJ, Tepper DE. The Cleveland Clinic Manual of Headache Therapy. In: Diagnosis of primary chronic daily headache. 2nd ed. New York: Springer Science, Business Media; 2011. p. 39-48.

2. Fumal A, Schoenen J. Tension-type headache: current research and clinical management. Lancet Neurol. 2008;7:70-83.

3. Ahmed F. Headache disorders: differentiating and managing the common subtypes. Br J Pain. 2012;6:124-32.

4. Bigal ME, Lipton RB. The epidemiology, burden, and comorbidities of migraine. Neurol Clin. 2009;27:321-34.

5. Buse DC, Rupnow MF, Lipton RB. Assessing and managing all aspects of migraine: migraine attacks, migraine-related functional impairment, common comorbidities, and quality of life. Mayo Clin Proc. 2009;84:422-35.

6. Magis D, Jensen R, Schoenen J. Neurostimulation therapies for primary headache disorders: present and future. Curr Opin Neurol. 2012;25(3):269-76.

7. Burt T, Lisanby H, Sackeim H. Neuropsychiatric applications of transcranial magnetic stimulation-a meta-analysis. Int J Neuropsychopharmacol. 2002; 5(1):73-103

8. Borckardt JJ, Smith AR, Reeves ST, Weinstein M, Kozel FA, Nahas Z, et al. Fifteen minutes of left prefrontal repetitive transcranial magnetic stimulation acutely increases thermal pain thresholds in healthy adults. Pain Res Manag. 2007;12(4):287-90.

9. Brighina F, Piazza A, Vitello Aloisio A, Palermo A, Daniele O, et al. Repetitive TMS of the prefrontal cortex in the treatment of chronic migraine. J Neurol Sci. 2004;227:67-71.

10. Lorenz J, Minoshima S, Casey KL. Keeping pain out of mind: the role of the dorsolateral prefrontal cortex in pain modulation. Brain. 2003;126:1079-91.

11. Kader AA, Fahmy E, Ahmad A, Labib A, Elmekkawy L. Conference abstract O206. The efficacy of repetitive transcranial magnetic stimulation in treating patients with chronic daily headache. Clin Neurophysiol. 2017.

12. International Headache Society. The International Classification of Headache Disorders. 2nd ed. Cephalalgia. 2004;24(1):9-160.

13. Santucci L, Story J. Transcranial magnetic stimulation for depression. J Psychiatry Neurosci. 2013;29:97-101.

14. Klirova M, Horacek J, Novak T, Cermak J, Spaniel F, Skrdlantova L, et al. Individualized rTMS neuro navigated according to regional brain metabolism ((18) FGD PET) has better treatment effects on auditory hallucinations than standard positioning of rTMS. Eur Arch Psychiatry Clin Neurosci. 2013;263(6):475-84.

15. Kader AA, Fahmy E, Ahmad A, Labib A, Elmekkawy L. The efficacy of repetitive transcranial magnetic stimulation in treating patients with chronic daily headache. Clin Neurophysiol. 2017;128(9):e244.

16. Lipton RB, Stewart WF, Diamond S, Diamond ML, Reed M. Prevalence and burden of migraine in the United States- data from the American Migraine Study II. Headache. 2001;41(7):646-57.

17. Bendtsen L, Evers S, Linde M, Mitsikostas DD, Sandrini G, Schoenen J. EFNS guideline on the treatment of tension-type headache - report of an EFNS task force. Eur J Neurol. 2010:17(11):1318-25.

18. Bendtsen L, Jensen R. Tension-type headache. Neurol Clin. 2009;27(2):525-35.

19. Kim BK, Chu MK, Lee TG. Prevalence and impact of migraine and tensiontype headache in Korea. J Clin Neurol. 2012;8(3):204-11.

20. Teneback CC, Nahas Z, Speer AM, Speer AM, Molloy M, Stallings L, et al. EChanges in prefrontal cortex and paralimbic activity in depression following two weeks of daily left prefrontal TMS. Neuropsychiatr Clin Neurosci. 1999:11:426-35.

21. Siebner HR, Rothwell J. Transcranial magnetic stimulation: new insight into representational cortical plasticity. Exp Brain Res. 2003;148:1-16.

22. Garcia JO, Grossman ED, Srinivasan R. Evoked potentials in large-scale cortical networks elicited by TMS of the visual cortex. J Neurophysiol. 2011; 106:1734-46. 
23. Brighina F, De Tommaso M, Giglia F, Scalia S, Cosentino G, Puma A, et al. Modulation of pain perception by transcranial magnetic stimulation of left prefrontal cortex. J Headache Pain. 2011;12(2):185-91.

24. Clarke BM, Upton AR, Kamath MV, Al-Harbi T, Castellanos CM. Transcranial magnetic stimulation for migraine: clinical effects. J Headache Pain. 2006;7: $341-6$.

25. Misra UK, Kalita J, Bhoi SK. High frequency repetitive transcranial magnetic stimulation (rTMS) is effective in migraine prophylaxis. Neurol Res. 2012; 34(6):547-51.

26. Kumar A, Mattoo B, Bhatia R, Kumaran S, Bahtia R. Neuronavigation based 10 sessions of repetitive transcranial magnetic stimulation therapy in chronic migraine: an exploratory study. Neurol Sci. 2020;24(1):131-9.

27. Kumar S, Singh S, Kumar N, Verma R. The effects of repetitive transcranial magnetic stimulation at dorsolateral prefrontal cortex in the treatment of migraine comorbid with depression: a retrospective open study. Clin Psychopharmacol Neurosci. 2018;16(1):62-6.

\section{Publisher's Note}

Springer Nature remains neutral with regard to jurisdictional claims in published maps and institutional affiliations.

\section{Submit your manuscript to a SpringerOpen ${ }^{\circ}$ journal and benefit from:}

- Convenient online submission

- Rigorous peer review

- Open access: articles freely available online

- High visibility within the field

- Retaining the copyright to your article

Submit your next manuscript at $\boldsymbol{\wedge}$ springeropen.com 